\title{
Cure monitoring of CFRP composites by dynamic mechanical analyser
}

\author{
Guillaume Kister*, Eleftheria Dossi
}

Centre for Defence Chemistry, Cranfield University, Defence Academy of the United Kingdom, Shrivenham SN6 8LA, UK

*Corresponding author. E-mail address: g.kister@cranfield.ac.uk (G. Kister).

\begin{abstract}
The cure characteristics of the carbon/MTM44-1 epoxy composite prepreg and the neat MTM44-1 epoxy resin are determined and monitored using a dynamic mechanical analyser (DMA). This study will also assess whether the simple containment device recommended by the DMA manufacturer for powder and gel is suitable for prepregs. The device is a disposable $0.1-\mathrm{mm}$ thick stainless steel pocket. The cure behaviour of the packaged materials is compared with that of the unpackaged prepreg. All the samples are cured following the manufacturer's $180^{\circ} \mathrm{C}$ isothermal schedule with heating rates of $2^{\circ} \mathrm{C} / \mathrm{min}$ and $5^{\circ} \mathrm{C} / \mathrm{min}$. The tests highlight that: (i) the cure of composite prepregs and resin can be monitored using a DMA; (ii) the stainless steel pocket does not influence the cure of the prepreg and resin; and (iii) the softening and melting of the uncured resin, the resin low viscosity state, and the subsequent gelation-vitrification-solidification of the resin are noticed with this method.
\end{abstract}

Keywords: Composite; Curing; DMA; Gelation; Vitrification; Dynamic mechanical analysis.

\section{Introduction}

Fibre-reinforced thermoset composites can be manufactured using different processing techniques such as resin transfer moulding (RTM) or autoclave. These processes require curing of the thermosetting matrix at ambient or elevated temperatures, which is a chemical reaction between a resin and a hardener or curing agent. This reaction is referred to as crosslinking reaction. The outcome will be the transformation of the resin from a liquid to a solid by the development of a threedimensional crosslink network. Since a chemical reaction takes place during this process, it is important to understand the behaviour cure state of the curing resin prior to commercialisation and manufacture of composite structures. Such knowledge will be useful for optimising of the manufacture process and for improving the prediction of the component's mechanical properties.

The behaviour of a curing resin or composite prepreg can be assessed using conventional analytical techniques that provide specific properties of the resin as a function of time and temperature. The most common technique is the differential scanning calorimetry (DSC) which is a thermal analysis technique measuring the thermal transitions occurring within the sample when it is heated, cooled or at isothermal temperature. The rein properties that can be extracted are the degree-ofcure, the heat flow during exotherm, the glass transition temperature and the melting and degradation temperatures [1-3].

Other techniques such as Fourier transformed infrared (FTIR) spectroscopy and rotating or oscillating rheometers can also be used to characterise the cure process of resins. The FTIR method consists of following the evolution of specific functional groups with time and temperature from which the degree-of-conversion of a functional 
group can be estimated [3-5]. On the other hand, the variation of the resin viscosity is monitored using rheometers during the initial stage of the cure (prior to gelation) $[6,7]$. thermosetting resins or composite prepregs is the dynamic mechanical analyser (DMA). This consists of measuring the material's viscoelastic properties, namely the storage and loss moduli and the damping factor (tanס) over a range of environmental and loading conditions. The majority of the application of DMA is to measure the glass transition temperature of solid or rubbery materials. However, this technique is also suitable to monitor the cure development and the gelation and vitrification transitions in thermosets. Cure monitoring of liquid resins with a DMA is achieved using either an inert impregnation braid or tape made of glass fibres, or a cup and probe or shear piston system $[5,8-10]$. The disadvantage with the former method is that low viscosity resins cannot be monitored without the risk of contaminating the oven and the drawback with the latter method is that the probe is trapped in the resin when gelation is reached; therefore information about the vitrification of the resin cannot be obtained. On the other hand, composite prepreg can be directly fitted between the DMA clamps in a single cantilever or torsion configuration $[1,12]$. Depending on the resin viscosity, the use of a thin nylon film enveloping the prepreg may be required in order to avoid the liquid resin from dripping inside the DMA oven [13]. Cure monitoring using the DMA highlighted the different transitions that a thermosetting resin encounters during the crosslinking reaction: the gelation - from a liquid to a rubber - and the vitrification - from a rubber to a glass material $[5,8,10,13,14]$.

The work presented in this paper focuses on assessing whether the use of a container device based on stainless steel pocket has an influence on the cure of a carbon/epoxy prepreg and an epoxy resin, which was monitored by dynamic mechanical analysis (DMA). The use of the pockets is a method recommended by the DMA manufacturer to determine the glass transition temperature of powders, gels and some viscous liquids. Such a device is economical, widely available and easy to set up in the DMA.

\section{Materials and methods}

The materials considered in this study were the unidirectional carbon fibre-reinforced MTM44-1 epoxy prepreg composite and the neat MTM44-1 epoxy resin, both supplied by Umeco Composites. The resin system is a one-part, high-performance, toughened, amine-based epoxy resin. The composite samples were prepared by stacking four layers of the prepreg (uncured ply thickness of $0.125 \mathrm{~mm}$; fibre volume fraction of $60 \%$ ) in the same direction using a roller to make unidirectional (UD) samples. The reinforcing fibres were all aligned along the length of the samples. The nominal dimensions of the uncured composite samples were $35.61 \times 6.18 \times 1.08 \mathrm{~mm}$ or 12.83 x $6.65 \times 1.07 \mathrm{~mm}$.

Regarding the neat epoxy resin, few milligrams (between 14.8 and $22.3 \mathrm{mg}$ ) of the material were extracted from a frozen solid block prior testing. Between tests, both materials were stored in a freezer according to the manufacturer's recommendations.

\section{Experimental processes}

\subsection{Dynamic mechanical analyser}

The viscoelastic properties of the curing materials were obtained by means of a Perkin Elmer DMA8000 dynamic mechanical analyser (DMA). The samples were subjected to a controlled sinusoidal displacement of $0.01 \mathrm{~mm}$ at the frequencies of 1,5 and 10 $\mathrm{Hz}$ in the single cantilever bending configuration. The storage modulus ( $\left.E^{\prime}\right)$, loss 
modulus (E") and damping factor (tanర్) were monitored as a function of temperature and time. the composite samples and $10 \mathrm{~mm}$ for the neat resin. Both materials were tested using the manufacturer's recommended cure schedule: heating from ambient temperature to $180^{\circ} \mathrm{C}$ at a rate of $2^{\circ} \mathrm{C} / \mathrm{min}$ or $5^{\circ} \mathrm{C} / \mathrm{min}$, followed by an isotherm at $180^{\circ} \mathrm{C}$ for up to $240 \mathrm{~min}$.

The composite samples were tested using two methods - with and without a pocket in order to assess the influence of such a container device on the cure. The stainless steel 'pockets' consisted of rectangular shims $(30 \times 14 \mathrm{~mm})$ that were cut from a 0.1 $\mathrm{mm}$ thick stainless steel plate (supplied by RS) and then folded lengthwise to form the pockets. The 12-mm long composite samples were then placed in the centre of the pockets. The tests with the unpackaged samples (without any pockets) were carried out with the 35-mm long composite samples that were just placed between the DMA clamps. However, in order to avoid bonding of the sample to the clamps, two 5-mm wide and $0.05-\mathrm{mm}$ thick stainless steel foils were wrapped around the composite sample to avoid direct contact of the curing resin with the clamps. The unidirectional composite samples were all placed/clamped in the same direction: with the carbon fibres aligned lengthwise i.e. from the fixed clamp to the vibrating clamp.

The stainless steel pockets were required to monitor the cure of the neat epoxy resin. The few milligrams of the resin were placed in the centre of the pockets. This test was performed to compare the cure characteristics of the resin with those of the packaged composite. Fig. 1 shows a stainless steel pocket and the packaged and unpackaged composite and resin samples.

\section{Fig. 1.}

\subsection{Rotational rheometer}

The viscosity of the neat MTM44-1 epoxy resin system was assessed using a rotary rheometer (C-VOR 150 Bohlin). Approximately $200 \mathrm{mg}$ of the resin were placed between two $25-\mathrm{mm}$ diameter aluminium discs spaced by a gap of $500 \mu \mathrm{m}$. The bottom disc was fixed whereas the top disc rotated. The resin system was tested under a single shear force of $50 \mathrm{~Pa}$. The resin was heated at a rate of $2^{\circ} \mathrm{C} / \mathrm{min}$ or $5^{\circ} \mathrm{C} / \mathrm{min}$ using an extended temperature cell up to the isothermal temperature of $180^{\circ} \mathrm{C}$. The initial test temperature was $50^{\circ} \mathrm{C}$ in order to soften the resin to obtain the required gap between the discs.

\section{Results and discussion}

\subsection{Cure monitoring of unpackaged composite}

The progress of the cure of the resin in the unpackaged unidirectional carbon/MTM441 epoxy composite can be followed by looking at the storage modulus (E'), the loss modulus ( $E$ ") and the damping factor (tanס) curves. Fig. 2 shows the variation of the storage modulus and temperature as a function of time and Fig. 3 presents the variation of $E^{\prime}$ and tan $\delta$ as a function of time. In these figures, the composite sample was heated up to the isothermal temperature at a rate of $5^{\circ} \mathrm{C} / \mathrm{min}$. Several important features of interest can be observed.

Fig. 2.

Fig. 3. 


\subsubsection{Softening of the matrix}

The storage modulus of the unpackaged composite sample decreased with increasing time or temperature until it reaches its lowest values at a temperature between $154^{\circ} \mathrm{C}$ and $165^{\circ} \mathrm{C}$ (between 26 and $29 \mathrm{~min}$ into the test; about 4 min before the start of the isotherm; label (2) in Fig. 2 and Fg. 3). Until about 15 min into the test (at a temperature of around $92^{\circ} \mathrm{C}$; label (1) in Fig. 2 and Fig. 3), the curves for the three frequencies of 1,5 and $10 \mathrm{~Hz}$ remained distinct but the frequency influence was gradually attenuated with increasing temperature. The decrease in E' and the attenuation of the frequency influence were the result of a change in the nature of the composite matrix that gradually softened with the increasing temperature until the resin lowest viscosity was reached between $154^{\circ} \mathrm{C}$ and $165^{\circ} \mathrm{C}$. The decrease in E' at the start of the heating was also observed in fibre-reinforced epoxy prepregs by Xie et al [10]. In addition, Stark et al. [15] also observed the initial influence of the frequency on the storage modulus and its subsequent attenuation with increasing temperature. This resin softening was interpreted by the authors as being the transition from the glassy to the rubbery state of the uncured resin at low temperature.

The storage modulus for each frequency curve initially decreased rapidly until the 5 and $10-\mathrm{Hz}$ curves became inseparable (at a temperature of about $65^{\circ} \mathrm{C} ; 9$ min into the test; label (1a) in Fig. 2 and Fig. 3). At a temperature below $65^{\circ} \mathrm{C}$, the values of $\mathrm{E}^{\prime}$ increased with increasing frequency. Above $65^{\circ} \mathrm{C}$, the storage modulus continued decreasing at a lower rate up to the temperature of around $95^{\circ} \mathrm{C}(15 \mathrm{~min}$ into the test; label (1) in Fig. 2 and Fig. 3), at which point the $1-\mathrm{Hz}$ curve commingled with the other two frequency curves. This highlighted the frequency dependence of the composite prepreg at low temperature when the uncured epoxy resin was in the glassy state. From $95^{\circ} \mathrm{C}$ to $154-165^{\circ} \mathrm{C}$ (between 26 and 29 min into the test; label (2) in Fig. 2 and Fig. 3), the storage modulus carried on decreasing until it reaches its lowest values. The unification of all three frequencies (label (1) in Fig. 3) was highlighted by a first minor peak in the tan $\delta$ curve, whereas the resin low viscosity was characterised by a trough following this minor peak.

The variation of the viscosity of the MTM44-1 epoxy resin and the cure temperature are presented in Fig. 4 as a function of time. The sample was heated to the isothermal temperature at a rate of $5^{\circ} \mathrm{C} / \mathrm{min}$. It was observed that the resin viscosity gradually decreased with increasing temperature until a low viscosity state was reached about 22 min into the test (this was obtained about 3 min before the start of the isotherm), after which the viscosity increased rapidly during gelation of the resin until solidification. The time corresponding to the low viscosity obtained by the rheometer was consistent with that for the low E' values measured by the DMA.

Fig. 4.

\subsubsection{Gelation of the matrix}

After reaching its lowest values, the storage modulus of the epoxy resin in the unpackaged composite increased with increasing temperature up to the isothermal temperature of $180^{\circ} \mathrm{C}$ and with increasing time. The $\mathrm{E}^{\prime}$ increase started at a moderate rate until about 40 min into the test (label (3) in Fig. 2 and Fig. 3) and then increased rapidly until 50 min into the test (label (4) in Fig. 2 and Fig. 3). Similar E' increases were observed in various studies when the isothermal temperature was reached $[13,15]$. These increases in $E^{\prime}$ corresponded to a change in the resin viscosity that was due to the start of the crosslinking reaction, which raises the average molecular 
weight of the resin close to infinity [2,9]. As a result, the resin underwent a transition from a liquid state to a solid state representing a change in the mechanical properties transition is referred to as gelation and the beginning of the frequency separation is considered as the gelation point. Fig. 2 and Fig. 3 also show that the start of the crosslinking reaction was independent of the test frequencies since no difference between the frequency curves was noticed. This is dissimilar to the observation made by Stark et al. [15] who noted a reduced deviation between the test frequencies. The acceleration of the crosslinking reaction at about 40 min (label (3) in Fig. 3) was highlighted by a second minor peak in the tan $\delta$ curve due to the change in the state of the resin.

\subsubsection{Vitrification of the matrix}

After gelation (about 50 min into the test; label (4) in Fig. 2 and Fig. 3), the E' values carried on increasing at an even higher rate before gradually levelling out between 84 and 110 min into the test (label (5) in Fig. 2 and Fig. 3). The reason for this rate increase is a change in the behaviour of the epoxy resin under DMA oscillation: it became once again dependent on the oscillation frequencies. This was noticed by the separation of the curves at the different frequencies in both Fig. 2 and Fig. 3. The higher the frequency, the higher the values of $E^{\prime}$. The difference between the frequency curves was gradually accentuated until levelling out.

The influence of the frequency on the resin was also noticed in the tanס where separation of the curves occurred at the start of the major peak (label (4) in Fig. 3). The peak maximum (label (5a) in Fig. 3) was reached during the increase of the storage modulus. The higher the frequency, the earlier the maximum peak was reached: at about $63 \mathrm{~min}$ and $71 \mathrm{~min}$ for the $10 \mathrm{~Hz}$ and $1 \mathrm{~Hz}$ frequencies, respectively. The end of the tan $\delta$ peak - characterised by a change in the decrease rate - occurred when the storage modulus values started to remain constant between 84 and $110 \mathrm{~min}$ depending on the frequency (label (5) in Fig. 3). This rapid increase in storage modulus and the tan $\delta$ peak corresponded to the vitrification of the resin - the time in the curing process when the resin solidifies. Vitrification indicates that the cure temperature is approaching or equalling the glass transition temperature of the fullycured resin due to the transition from a rubbery to a glassy state $[5,10]$. However, vitrification does not necessarily indicate the end of the formation of the crosslink network.

\subsubsection{Solidification of the matrix}

From 110 min into the test, the storage modulus remained relatively constant up to the completion of the test. The frequency curves for E' remained distinct up to about 190 min into the test (label (6) in Fig. 2 and Fig. 3) at which time they were inseparable until the end of the test. This unification of the frequency curves also occurred in the tanס and this was marked by the curves levelling out. Varley et al. [8] stipulated that when the storage modulus levelled out beyond vitrification, the crosslinking reaction was completed. However it does not explain the frequency dependence of the storage modulus and tanס. A possible explanation is that the separation of the frequency curves could be due to the presence of localised 'volumes' of uncured resin between the nearly-completed principal crosslink network, consequently locking the volumes of resin in a solid structure. The resin in these volumes continued to react until saturation of crosslinks at about $190 \mathrm{~min}$. The presence of these resin volumes had a localised effect on the frequency-dependent storage modulus but no real influence on the 
overall modulus of the whole sample (E' relatively constant whatever the frequency). From 190 min, the sample became an elastic solid material without any 'viscous'

\subsubsection{Comparison between the heating rates of $2^{\circ} \mathrm{C} / \mathrm{min}$ and $5^{\circ} \mathrm{C} / \mathrm{min}$}

Heating the composite samples at a heating rate of $2^{\circ} \mathrm{C} / \mathrm{min}$ prior to reaching the isothermal temperature had no real influence on the curing behaviour of the epoxy resin. Similar variations of the storage modulus and tanঠ curves were observed but the times at which the glass-rubber transition of the uncured resin occurred and the low viscosity was reached, were increased by $55 \%$. On the other hand, the temperatures at which these features occurred were lower by about $5^{\circ} \mathrm{C}$ compared with those during a heating rate of $5^{\circ} \mathrm{C} / \mathrm{min}$. The features observed during the isotherm took place approximately 9 min earlier in the samples heated at a rate of $2^{\circ} \mathrm{C} / \mathrm{min}$.

\subsection{Cure monitoring of packaged composite and resin}

One of the advantages of the MTM44-1 epoxy resin is that it remains relatively viscous even when the lowest viscosity threshold is reached; therefore, there was little risk of damage to the DMA instrument from dripping resin. However, should a resin becomes too liquid during cure, a container device such as a stainless steel pocket or nylon film has to be used to assess the curing properties of the resin by the DMA technique [13]. However, such a method could attenuate or even hide some of the curing characteristics of the resin. For this reason, the curing behaviour of the unidirectional carbon/MTM44-1 epoxy prepreg was monitored when the sample was placed in a stainless steel pocket and then compared with the unpackaged composite samples. The cure of the neat epoxy resin was also monitored using a pocket and the resin behaviour was compared with that of the packaged composite.

Isothermal tests on the stainless steel pockets alone confirmed that the storage modulus decreased linearly by less than $5 \%$ with increasing temperature, the damping factor remained constant and the frequency did not have any influence on the pocket behaviour. Therefore, the pockets would not have any influence on the visco-elastic behaviour of the curing samples. Consequently, any variations in the storage modulus and tanঠ curves would be attributed to the sample.

The curing characteristics of the carbon/epoxy prepreg and the neat epoxy resin contained in stainless steel pockets are shown in Fig. 5 and Fig. 6 respectively, where the storage modulus ( $\left.E^{\prime}\right)$ and the damping factor $(\tan \delta)$ are plotted as a function of the test duration (the heating rate was $2^{\circ} \mathrm{C} / \mathrm{min}$ ).

Fig. 5.

Fig. 6.

Most of the curing characteristics observed in the unpackaged composite and described in the previous section can also be noticed in the composite and neat resin contained in stainless steel pockets. The glass to rubber transition of the softening uncured epoxy resin was noticed by a change in the slope of the decreasing storage modulus and tan $\delta$ values at the temperature of about $65^{\circ} \mathrm{C}$ (label (1a) in Fig. 5 and Fig. 6). At that temperature, the frequency curves were generally inseparable in most of the packaged composite and neat resin sample heated at a rate of $2^{\circ} \mathrm{C} / \mathrm{min}$ and in all the samples heated at $5^{\circ} \mathrm{C} / \mathrm{min}$. In the other samples heated at $2^{\circ} \mathrm{C} / \mathrm{min}$, the 
unification of the $1 \mathrm{~Hz}$ curve with the other two frequency curves was seen to take place at about $95^{\circ} \mathrm{C}$ (label (1) in Fig. 5 and Fig. 6). It can therefore be assumed that softening resin.

The low viscosity regime highlighted by the lowest E' values in both packaged samples was reached about 3 min before and about 4 min after reaching the isothermal temperature when the heating rate was $2^{\circ} \mathrm{C} / \mathrm{min}$ and $5^{\circ} \mathrm{C} / \mathrm{min}$, respectively (label (2) in Fig. 5 and Fig. 6). This low viscosity state was difficult to observe in the tanঠ curves: a slight decrease in the values could be noticed after the tan $\delta$ remained constant during the resin softening process. For a specific heating rate, the values of the lowest storage modulus and the time when these values were reached were similar for both packaged materials. On the other hand, the lowest E' values in the packaged samples were obtained on average 9 min after those in the unpackaged samples whatever the heating rate, which could be the result of the thermal effect of the metallic pockets.

In the packaged samples, the gelation of the epoxy resin was highlighted by the increase of the storage modulus values from the state of low viscosity (label (2) in Fig. 5 and Fig. 6) to the separation of the frequency curves defined as the gelation point (about 96 min into the test; label (4) in Fig. 5 and Fig. 6). The start of the crosslinking reaction was not obvious from the damping factor curves but the frequency dependence of the polymerising resin was marked by a rapid increase in the tan $\delta$ values and by a separation of the different frequency curves (label (4) in the figures). The frequency separation in the packaged samples occurred on average between 3 to 7 min after the separation in the unpackaged samples; however, no time difference was observed between both packaged materials.

Vitrification of the resin in the packaged samples was highlighted by the rapid increase in the storage modulus values and by the large tanס peak (labels (5) in Fig. 5 and Fig. 6). At that stage in the cure, the behaviour of the composite and resin was dependent on the DMA oscillation frequency. The end of vitrification is generally marked by the levelling of the E' values and by a change in the tan $\delta$ decrease rate, both occurring at the same time. However, this characteristic was only observed in the packaged composite and not in the packaged resin. This could be attributed to the larger volume of resin present in the packaged resin samples than in the packaged composites. The gradual increase of E' or gradual decrease of tanס in the neat resin samples could be attributed to a further opening of the unreacted epoxide rings that crosslinked with some remaining amine hardener. The larger volume of packaged neat resin could also be the reason for the maximum tan $\delta$ being reached 20 min later than that for the packaged composite. However, no time delay was observed between the packaged and unpackaged composite samples.

Solidification of the epoxy resin (label (6) in Fig. 5) was only observed in the packaged composite samples by a unification of the frequency curves of the storage modulus and by the damping factor curves remaining constant from about 190-230 min into the test. Unification of the frequency curves in the neat resin was not observed within the test duration; however, the difference between the frequency curves was reducing with time. The unification of the frequency occurred at the same time in both the packaged and unpackaged composite samples.

\subsection{Comparison between unpackaged and packaged samples}

The use of stainless steel pockets did not greatly influence the visco-elastic behaviour of the curing composite samples. All the cure characteristics described in the previous subsections (those labelled in the figures) were observed in the unpackaged and 
packaged samples. However, the full solidification of the neat epoxy resin did not seem to occur during the test duration as the behaviour of the solid resin remained influenced by the test frequencies.

A thermal/time delay was observed between the packaged and the unpackaged samples as far as the resin low viscosity state and the gelation point were concerned. These states were reached in the packaged samples between 3 to 9 min after the unpackaged samples. This means that the pockets slightly delayed the melting and crosslinking of the resin during the temperature increase and at the beginning of the isotherm, which could be the result of the relatively high thermal conductivity and diffusivity properties of the stainless steel.

During the melting of the resin, it was observed that the tan $\delta$ curves in the packaged samples were flatter than in the unpackaged samples. The peaks and troughs associated with the softening of the resin (label (1)), the resin lowest viscosity (label (2)) and the acceleration of the crosslinking reaction (label (3)) were only noticed in the unpackaged composite samples. This means that the stainless steel pockets might be hiding subtle variations in the resin behaviour due to the material's stiffness. The other influence of the stainless steel pockets can be seen during the glass to rubber transition of the uncured resin (at the start of the heating process). In all the unpackaged samples, the dependence of the resin to the test frequencies was clearly observed by the separation of the frequency curves until unification when the resin was liquid enough (labels (1) and (1a)). However, the distinction between the frequency curves was more difficult to make in most of the packaged composite and resin samples (after label (1a)). The reason could be attributed to the stiffness of the pockets compared to that of the softening unpackaged composite at the start of the material cure schedule.

Another difference is in the amplitude of the storage modulus values between the start of the test at $20^{\circ} \mathrm{C}$ and the temperature of the lowest viscosity (between $160^{\circ} \mathrm{C}$ and $180^{\circ} \mathrm{C}$ ). The $\mathrm{E}^{\prime}$ amplitude was on average 6 times larger in the unpackaged composite samples than in the packaged composite. For example, the amplitudes for the samples heated with a rate of $2^{\circ} \mathrm{C} / \mathrm{min}$ and oscillated at the frequency of $1 \mathrm{~Hz}$ were $1.21 \mathrm{GPa}$ for the unpackaged sample and $0.25 \mathrm{GPa}$ for the packaged composite. Moreover, no difference was observed between the E' amplitudes of both packaged resin and composite samples. This showed that the stiffness of the pockets influenced the storage modulus values of the tested material. The amplitude of the storage modulus in all the samples was found to be reduced by an average of $14 \%$ when the heating rate was increased from $2^{\circ} \mathrm{C} / \mathrm{min}$ to $5^{\circ} \mathrm{C} / \mathrm{min}$. This could indicate that lower viscosity might be achieved when the heating rate is low, which would allow better impregnation of the reinforcing fibres in the composites.

Stainless steel pockets of different thicknesses were also used to evaluate their influence on the cure of the epoxy resin. The other two thicknesses were $0.05 \mathrm{~mm}$ and $0.2 \mathrm{~mm}$. It was observed that (i) the thinner the pocket, the noisier the DMA curves, and (ii) the thinner the pocket, the shorter the time/temperature delay for the low viscosity state and the gelation point. It was found that a $0.1-\mathrm{mm}$ thick pocket was the best compromise as far as sample handling and cure monitoring were concerned.

\section{Conclusions}

The isothermal cure of a carbon/epoxy composite prepreg and the neat epoxy resin was monitored using the dynamic mechanical analyser (DMA) method. Some of the prepreg samples and the neat resin were packaged in stainless steel pockets and their visco-elastic behaviour was compared with that of the unpackaged composite samples. It was found that (i) the cure characteristics such as the glass-rubber 
transition of the uncured resin, the low viscosity state and the gelation-vitrificationsolidification of the resin were detected by the DMA technique, (ii) the pockets did not the resin cure characteristics. The curing differences between the packaged and unpackaged samples were found to be (i) a slight time/temperature delay due to the thermal diffusivity of the stainless steel, and (ii) a reduction in the storage modulus amplitudes and a suppression of the subtle tano variations during softening of the resin due to the stiffness of the pocket material.

\section{Acknowledgements}

The authors would like the acknowledge Umeco Composites in Derbyshire, UK, for the free supply of the neat MTM44-1 epoxy resin.

\section{References}

1. W. Stark, M. Jaunich, J. McHugh. Cure state detection for pre-cured carbon-fibre epoxy prepreg (CFC) using temperature-modulated differential scanning calorimetry (TMDSC). Polym Test 2013;32:1261.

2. S.R White, P.T Mather, M.J Smith. Characterisation of the cure-state of DGEBADDS epoxy using ultrasonic, dynamic mechanical and thermal probes. Polym Eng Sci 2002;42:51.

3. J. Zhang, T. Li, H. Wang, Y. Liu, Y. Yu. Monitoring extent of curing and thermalmechanical property study of printed circuit board substrates. Microelectron Reliab 2014;54:619.

4. B. Degamber, G.F Fernando. Fibre optic dilato-spectroscopic sensor: simultaneous thermal, spectral, and physical analyses of materials. Smart Mater Struct 2006;15:1054.

5. B. Zhou, M. Shen, Q. Wang, Q. Chen. Kinetic study of polysulfide-acrylate click reaction by DEA and DMA. Polym Adv Technol 2011;22:2374.

6. J.H Daly, D. Hayward, R.A Pethrick. Prediction of the rheological properties of a curing thermoset system. Macromolecules 2013;46:3621.

7. S. Mortimer, A.J Ryan, J.L Stanford. Rheological behaviour and gel-point determination for a model Lewis acid-initiated chain growth epoxy resin. Prediction of the rheological properties of a curing thermoset system. Macromolecules 2001;34:2973.

8. R.J Varley, J.H Hodgkin, D.G Hawthorne, G.P Simon. Toughening of a trifunctional epoxy system. II. Thermal characterisation of epoxy/amine cure. J Appl Polym Sci 1996;60:2251.

9. A. Cadenato, J.M Salla, X. Ramis, J.M Morancho, L.M Marroyo, J.L Martin. Determination of gel and vitrification times of thermoset curing process by means of TMA, DMTA and DSC techniques TTT diagram. J Therm Anal 1997;49:269.

10. M. Xie, Z. Zhang, Y. Gu, M. Li, Y. Su. A new method to characterize the cure state of epoxy prepreg by dynamic mechanical analysis. Thermochim Acta 2009;487:8.

11.W. Stark. Investigation of the curing behaviour of carbon fibre epoxy prepreg by dynamic mechanical analysis DMA. Polym Test 2013;32:231.

12. K.P Menard. In Dynamic mechanical analysis. A practical introduction. Second Edition. CRC Press Taylor and Francis. 2008.

13. J. Jakobsen, M. Jensen, J.H Andreasen. Thermo-mechanical characterisation of in-plane properties of CSM E-glass epoxy polymer composite materials - Part 2: Young's modulus. Polym Test 2013;32:1417.

14. B. Bilyeu, W. Brostow, K.P Menard. Separation of gelation from vitrification in curing of a fibre-reinforced epoxy composite. Polym Compos 2002;23:1111. 
15. W. Stark, M. Jaunich, J. McHugh. Carbon-fibre epoxy prepreg (CFC) curing in an autoclave analogue process controlled by dynamic mechanical analysis (DMA). Polym Test 2013;32:1487. 


\section{Figure captions}

Fig. 1. Configurations for the composite and resin samples.

Fig. 2. Storage modulus (log scale) and temperature (linear scale) of the curing carbon/epoxy composite prepreg as a function of time (heating rate of $5^{\circ} \mathrm{C} / \mathrm{min}$ ).

Fig. 3. Storage modulus and damping factor (log scales) of the curing carbon/epoxy prepreg as a function of time (heating rate of $5^{\circ} \mathrm{C} / \mathrm{min}$ ).

Fig. 4. Viscosity variation (log scale) of the MTM44-1 resin measured using a rheometer (heating rate of $5^{\circ} \mathrm{C} / \mathrm{min}$ ).

Fig. 5. Storage modulus and damping factor (linear scales) of a curing carbon/epoxy composite in a stainless steel pocket as a function of time (heating rate of $2^{\circ} \mathrm{C} / \mathrm{min}$ ).

Fig. 6. Storage modulus and damping factor (linear scales) of a curing neat epoxy resin as a function of time (heating rate of $2^{\circ} \mathrm{C} / \mathrm{min}$ ). 
Stainless steel pocket
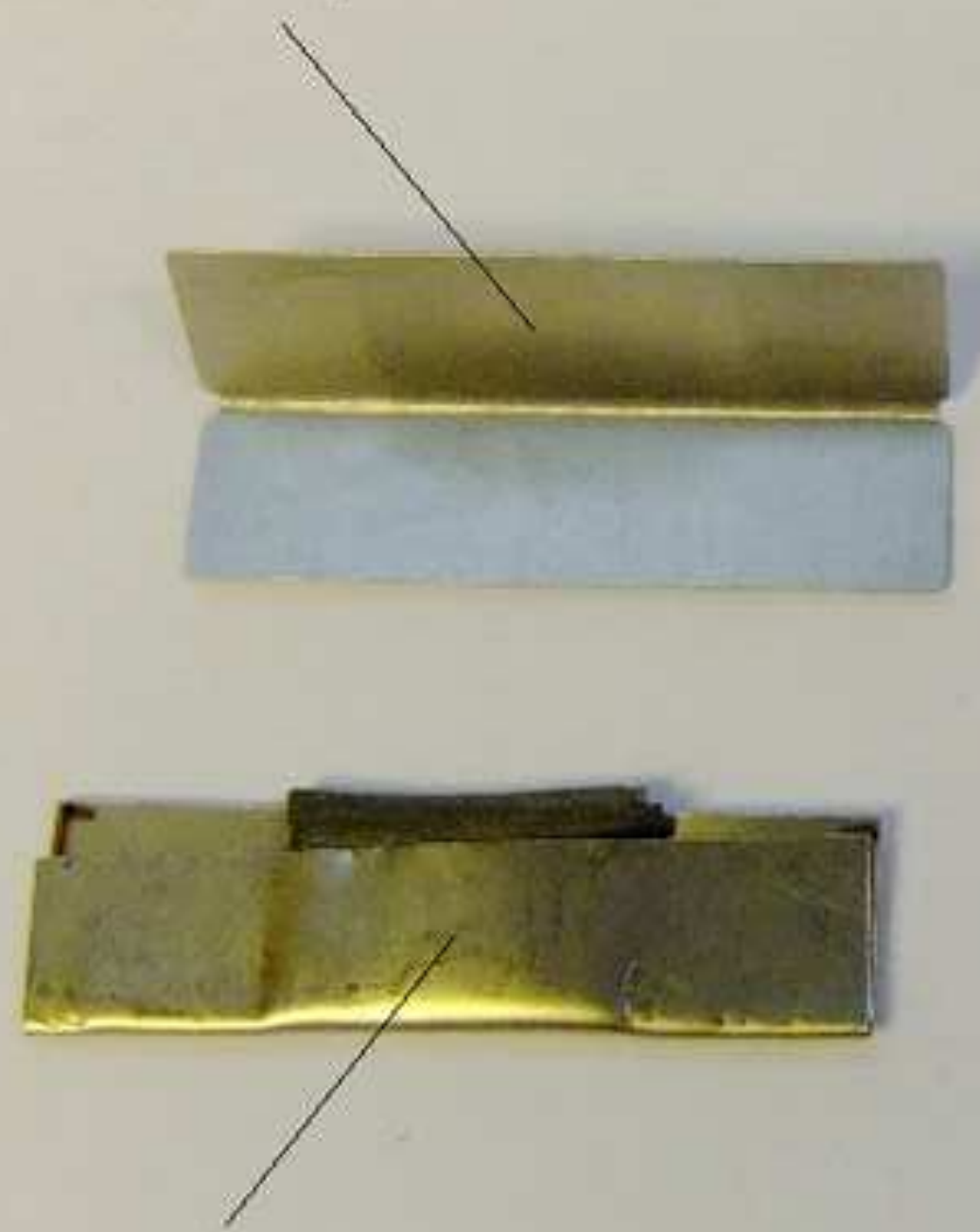

UD carbon/epoxy composite in a pocket
Neat MTM44-1 epoxy resin in a pocket
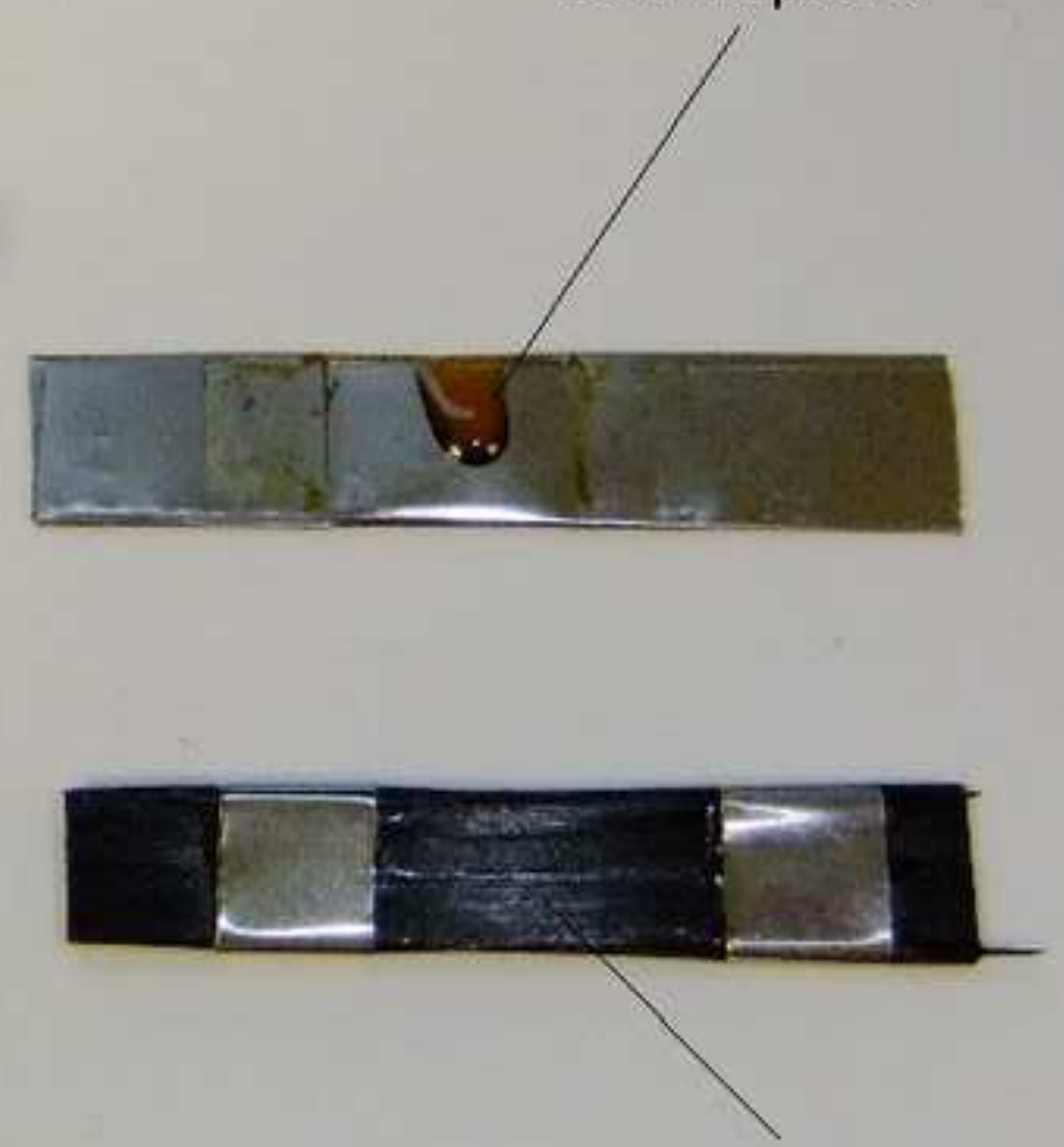

UD carbon/epoxy composite with stainless steel foils 


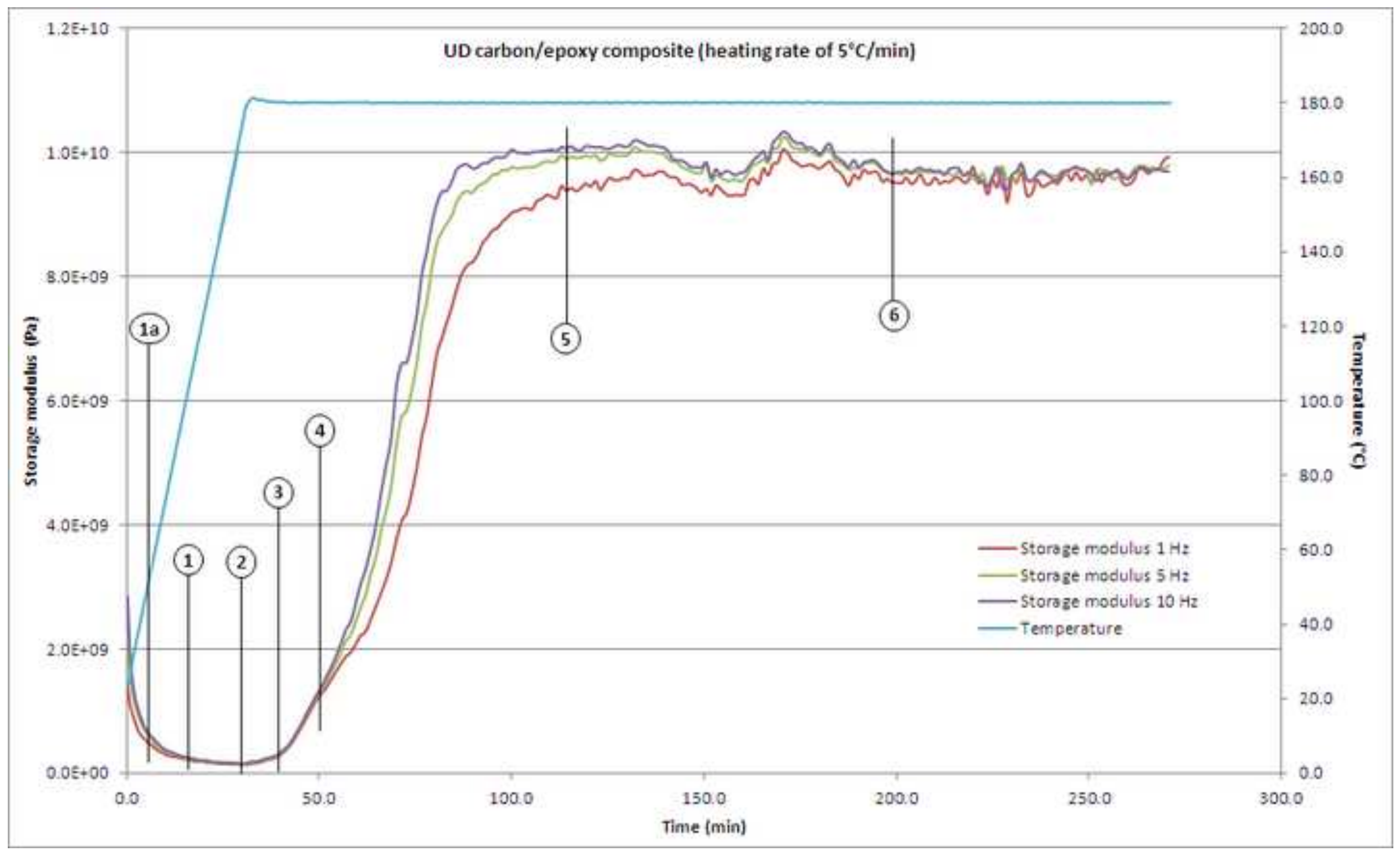




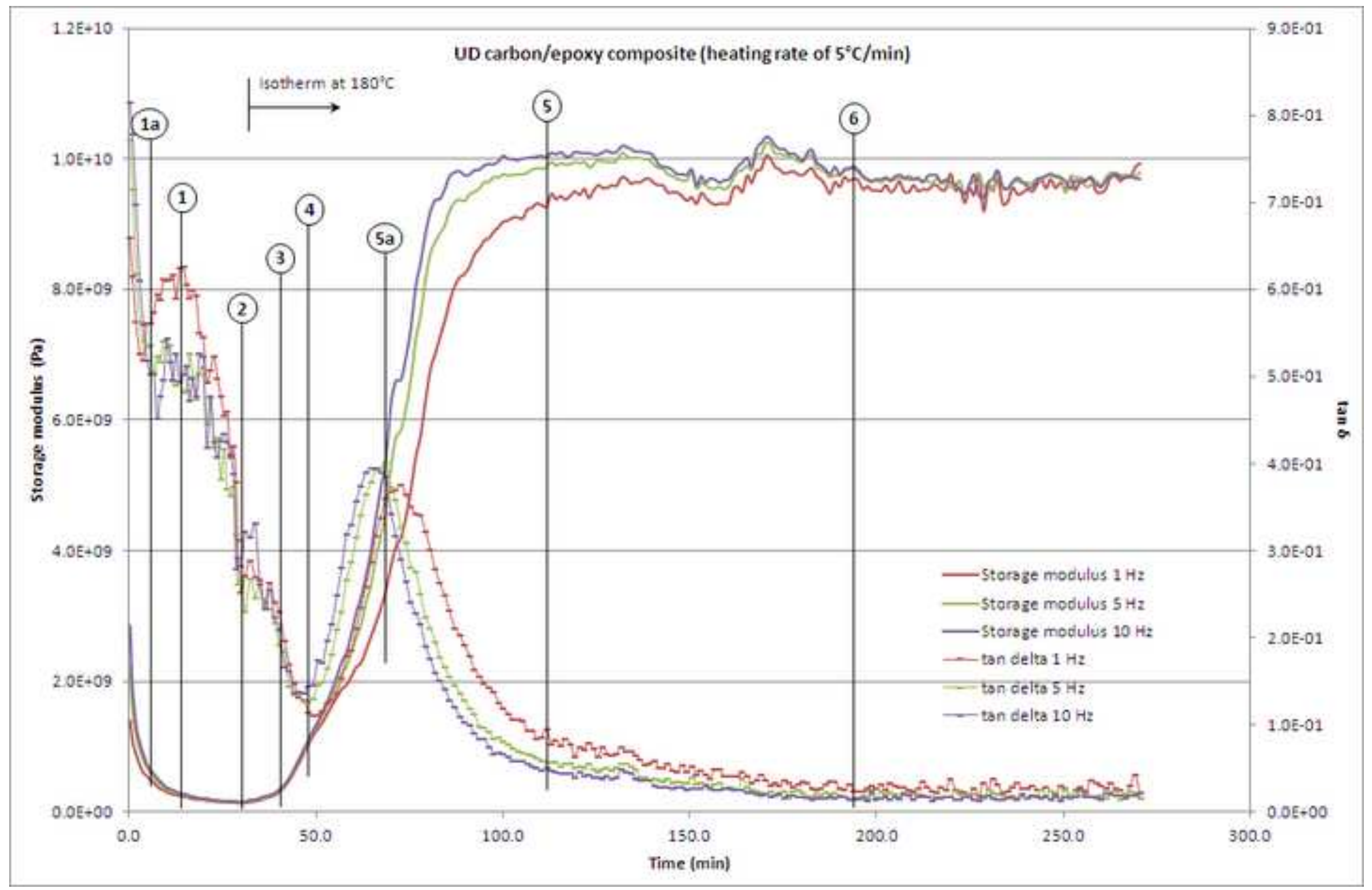




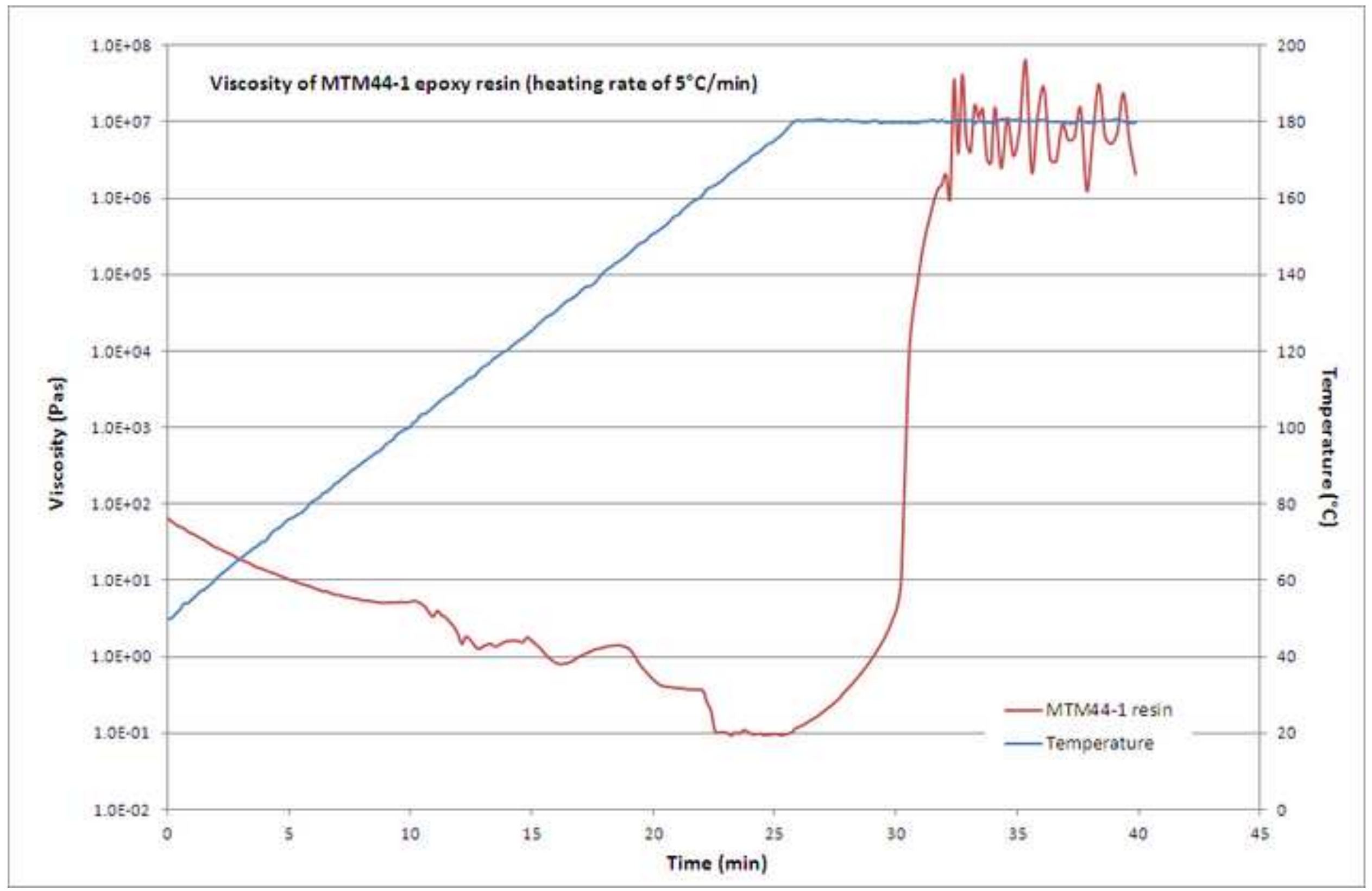




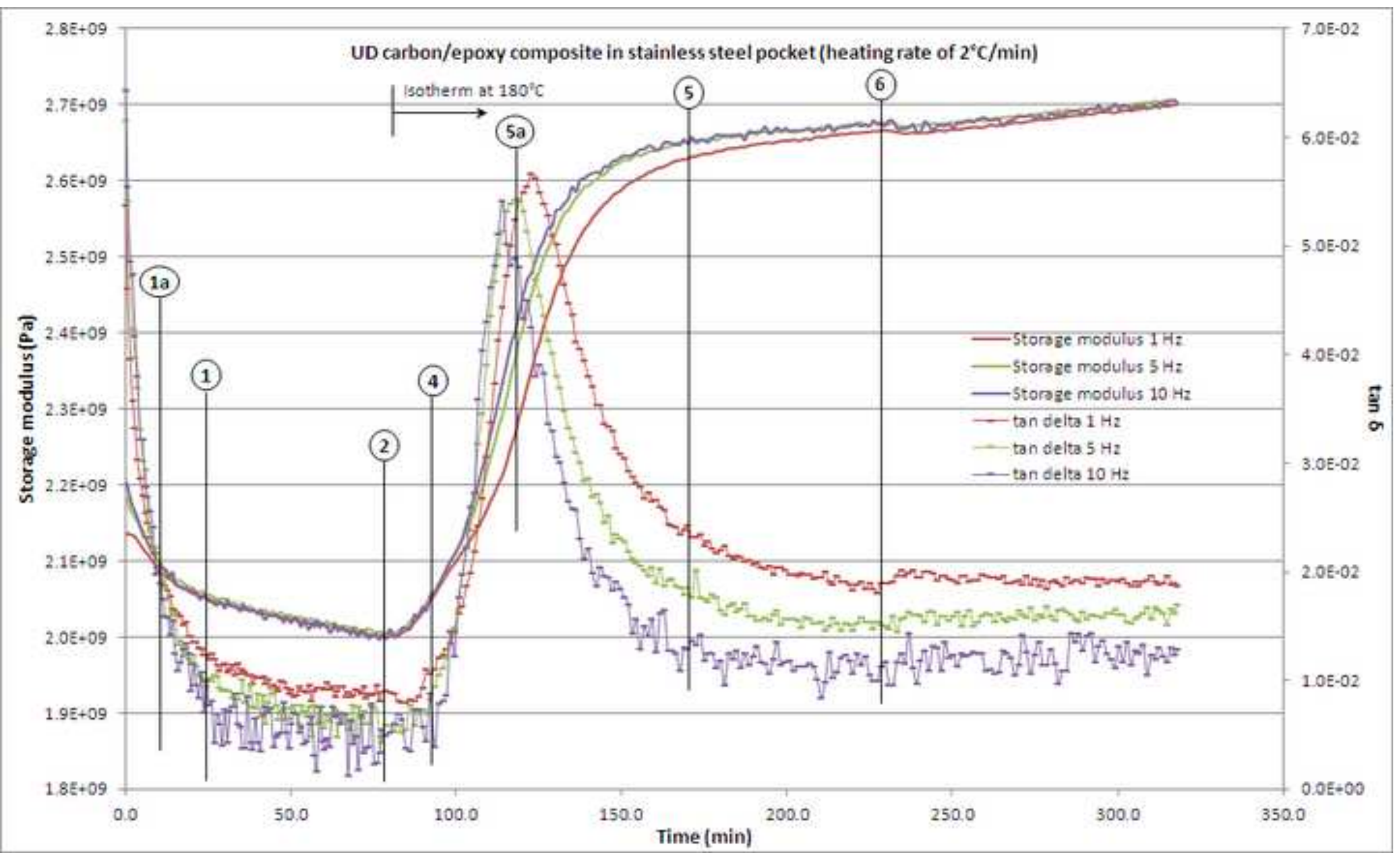




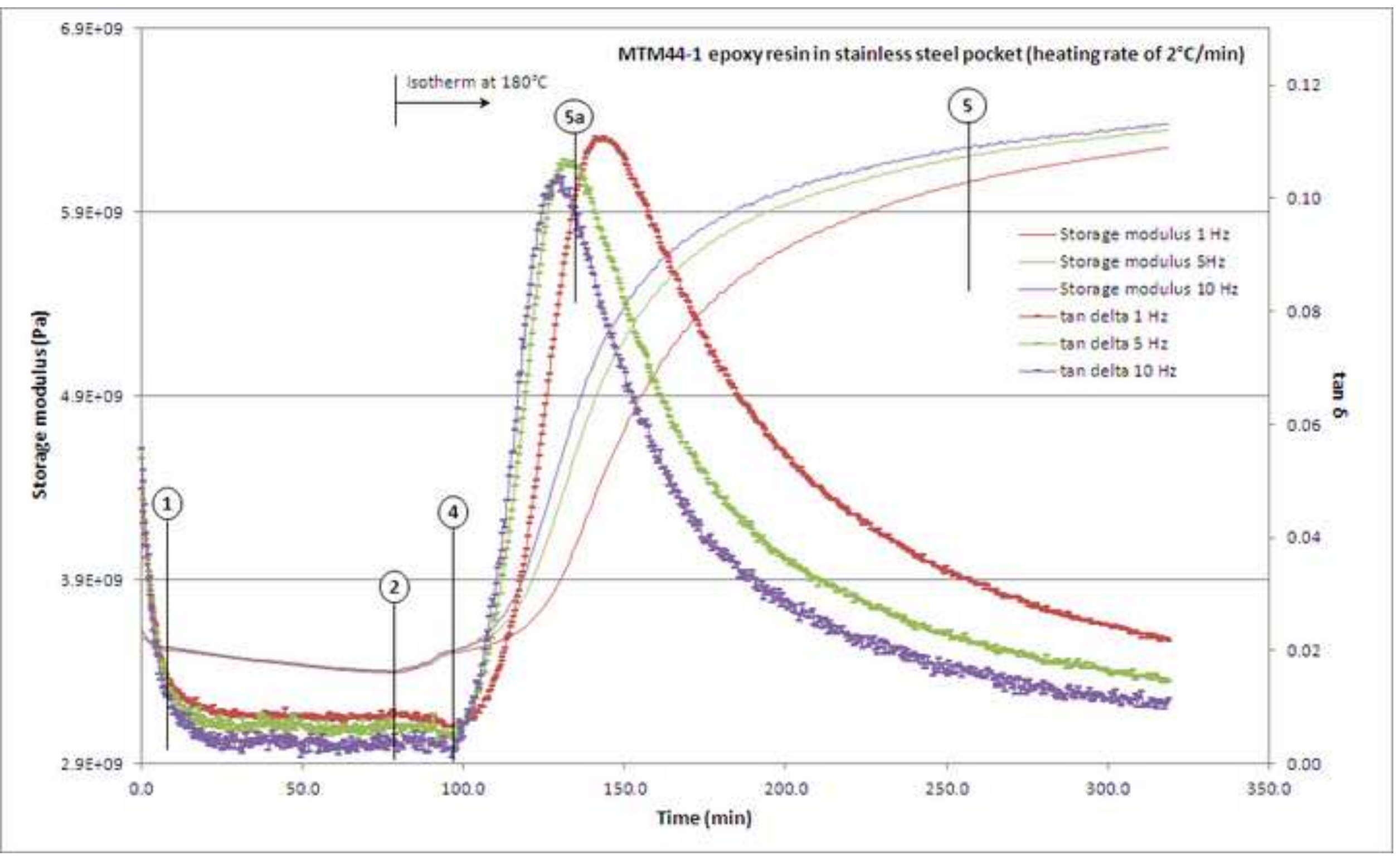

